left in no doubt where the author's sympathies lie, and why - "Homology is a statement about pattern, and should not be conflated with a concept about processes and mechanisms". Before I read the book, I thought this was exactly the opposite of the truth. Now I am not so sure. I appreciate more clearly that, to quote another of Hall's quotes, "Among evolutionary biologists, homology has a firm reputation as an elusive concept" (Wagner, 1989).

For anyone with an interest in the subject, Hall's book is a valuable guide to who thought what, and where to find it. But don't use it as a primer.

Michael E. Akam is in the Wellcome/CRC Institute of Cancer and Developmental Biology, Tennis Court Road, Cambridge CB2 1QR, UK.

\section{Time warp}

Oliver Penrose

Time's Arrow: The Origins of Thermodynamic Behaviour. By Michael C. Mackey. Springer: 1992. Pp. 175. DM98, £40, \$49.

THE second law of thermodynamics always arouses interest because it is the only widely applicable law of physics that is not symmetric under time reversal, and so singles out a particular direction of time. The law states that if a physical system is thermally isolated (that is, if no heat enters or leaves the system), the thermodynamic quantity known as entropy must increase or stay constant. The law therefore singles out the time direction of irreversible physical processes as the direction of increasing entropy.

For example, imagine two cylinders of gas joined by a narrow pipe containing a valve that is at first closed, then open for a short time, then closed again. Somebody measures how the gas is distributed between the cylinders before the valve opens and after it closes, writes the results on two cards, and asks you to tell which card describes the earlier state and which the later. One way of solving the puzzle would be to use the second law. It is not difficult to show that (other things being equal) the more evenly the gas is distributed between the two containers, the greater the entropy; so the state with the gas more evenly distributed between the two cylinders is the one that occurred later.

When the second law was originally put forward by the founders of thermodynamics, it must have seemed completely mysterious. Unlike the other concepts of thermodynamics such as energy, temperature, pressure and so on, entropy had no counterpart in mechanics or everyday experience. But in 1872 Ludwig Boltzmann discovered one of the jewels of nineteenth-century physics, a precise quantitative relation giving the entropy of a gas in terms of mechanical quantities, namely the distribution of positions and velocities of its molecules. He showed, in his famous ' $H$ theorem', that if the gas is not in equilibrium then collisions will increase the entropy until finally a state of equilibrium is reached.

Around the turn of the century, J. Willard Gibbs discovered another important formula connecting entropy with mechanical quantities. Unlike Boltzmann's formula for the entropy of a gas, that of Gibbs applies to liquids and solids as well; but it pays for this greater generality by being applicable unambiguously only to systems that are in equilibrium.

In real life - or even in the physics laboratory - we frequently encounter objects that are neither gases nor in equilibrium. One would like to have a 'time's arrow' covering this more general case too - that is, a formula for the entropy of a non-gaseous nonequilibrium system that can be shown to have the property of non-decrease in time if the system is thermally isolated. Neither of the formulas so far mentioned will do: the Gibbs formula gives an entropy that for an isolated system of molecules is easily shown to stay constant in time rather than to increase, and the Boltzmann formula also does not have the desired non-decrease property if the system is not a gas.

The title of this book leads one to expect a contribution to the solution of this problem, but unfortunately these hopes are soon dashed. The discussion is based on what the author calls the "Boltzmann-Gibbs" entropy, but in the terminology used above it is just the Gibbs entropy. His claim in Chapter 1 to prove that this is essentially the only possible definition is belied by the existence of Boltzmann's definition, which is certainly different. The author notes that the entropy, as he has defined it, is constant for systems whose time evolution is governed by differential equations such as the laws of newtonian mechanics. Because he wants the entropy to increase with time, the obvious conclusion would be that the wrong definition of entropy has been chosen and that it should be changed, but he decides instead to change the law of time evolution. The change he makes is to assume that the laws of motion obeyed by the particles constituting an isolated system are non-invertible: that is, that the same dynamical state at a given time can be reached from two or more different dynamical states at some earlier time.
Because the generally accepted laws of motion for such a system of particles do not have this property, his assumption is a departure from what we know about physical reality.

The rest of the book is an exploration of the consequences of this unphysical assumption. The hypothetical world where this assumption holds contains, indeed, many interesting things, and the author guides us around it skilfully. Unlike some mathematical authors, he does not write as though his prime objective is to show how clever he is; he provides plenty of examples to illustrate his theorems and definitions, and clear summaries at the end of each chapter. All the book needs to make it worthy of recommendation as an introduction to this enjoyable branch of mathematics is a health warning like the one on the tobacco advertisements: "Reading this book can damage your understanding of physical reality".

Oliver Penrose is in the Department of Mathematics, Heriot-Watt University, Riccarton, Edinburgh EH14 4AS, UK.

\section{New in paperback}

Understanding the Present: Science and the Soul of Modern Man by Bryan Appleyard. This book was the cause of considerable controversy when first published in the United Kingdom last year. The author's central argument is that "Science is not a neutral or innocent commodity .... a convenience. Rather, it is spiritually corrosive, burning away ancient authorities and traditions". Nature's commentators were not convinced for reviews see Nature 356, 729 and 357, 29; 1992. Picador, £6.99. (US hardback edition recently published by Doubleday, $\$ 23.50$.)

Vital Circuits: On Pumps, Pipes and the Workings of Circulatory Systems by Steven Vogel. In a review in Nature 358, 720 (1992), David Weatherall wrote that " $[$ Vogel] leads us through the functions and regulation of the heart and circulation with extraordinary clarity and lightness of touch. It is difficult to find fault with this eccentric and lively account". Oxford University Press, \$12.95.

\section{Spring Books}

Next week's issue (15 April) contains Nature's Spring Books supplement.

Among featured reviewers will be Steve Blinkhorn on the psychology of interrogations, confessions and testimony; Fred Hoyle on the works of Copernicus and Kepler; L. Sprague de Camp on polar mythology; John Mollon on Vincent van Gogh, chemicals and creativity; and Alison Jolly on chimpanzees and humans. 